\title{
Counting targets with mobile sensors in an unknown environment
}

\section{Report}

Author(s):

Suri, Subhash; Gfeller, Beat; Mihalák, Matúš; Vicari, Elias; Widmayer, Peter

Publication date:

2007

Permanent link:

https://doi.org/10.3929/ethz-a-006809894

Rights / license:

In Copyright - Non-Commercial Use Permitted

Originally published in:

Technical Report / ETH Zurich, Department of Computer Science 571 


\title{
Counting Targets with Mobile Sensors in an Unknown Environment
}

\author{
ETH Technical Report 571
}

\author{
Subhash Suri* \\ Department of Computer Science \\ University of California \\ Santa Barbara, USA 95106 \\ Beat Gfeller, Matúš Mihalák, Elias Vicarił Peter Widmayer ${ }^{\dagger}$ \\ Institute of Theoretical Computer Science, \\ ETH Zurich, 8092 Zurich, Switzerland
}

October 9, 2007

\begin{abstract}
We consider the problem of counting the number of indistinguishable targets using a simple binary sensing model. Our setting includes an unknown number of point targets in a (simply- or multiply-connected) polygonal workspace, and a moving point-robot whose sensory input at any location is a binary vector representing the cyclic order of the polygon vertices and targets visible to the robot and the presence of walls between consecutive vertices. In particular, the sensing model provides no coordinates, distance or angle measurements. We investigate this problem under two natural models of environment, friendly and hostile, which differ only in whether the robot can visit the targets or not, and under three different models of motion capability.

In the friendly scenario we show that the robots can count the targets, whereas in the hostile scenario we prove that no $(2-\varepsilon)$-approximation is possible, for any $\varepsilon>0$ and we show a 2-approximation algorithm. Further we present a class of polygons recognizable by our robots where the problem can be exactly solved. Next we consider two, slightly more powerful robots ans show that they can count the targets exactly in any polygon.
\end{abstract}

*Work done while the author was a visiting scholar at the Institute of Theoretical Computer Science, ETH, Zurich.

${ }^{\dagger}$ Work partially supported by the National Competence Center in Research on Mobile Information and Communication Systems NCCR-MICS, a center supported by the Swiss National Science Foundation under grant number $5005-67322$. 


\section{The Problem and the Model}

Simple, small and inexpensive computational and sensing devices are currently at the forefront of several research areas in computer science. These devices promise to bring computational capabilities into areas where previous approaches (usually consisting of complex and bulky hardware) are not feasible or costeffective. Such devices are being successfully used in various monitoring systems, military tasks, and other information processing scenarios. Their main advantages are quick and easy deployment, scalability, and cost-effectiveness. However, in order to realize the full potential of these technologies, many new and challenging research problems must be solved, because the classical schemes designed for centralized and desktop computational hardware are inapplicable to the lightweight and distributed computational model of sensor nodes. The inherent limitations of the systems based on these simple devices have inspired the research community to consider the computation with a minimalistic view of hardware complexity, sensing and processing, energy supply, etc.

In this paper we use such a minimalistic approach in the area of mobile sensors - simple robots. We consider and define robots of unsophisticated sensing and mobile capabilities and investigate their computational power on an elementary yet natural problem of counting objects of interest in the robots' environment. We model the environment by a polygon $P$ (simply or multiplyconnected) in the plane and the objects of interest, namely, targets are modeled as a set of points inside $P$.

A robot is a (moving) point, equipped with a simple camera that can sense just the combinatorial features of the surrounding. In particular, the robot can see a vertex of $P$ or a target, can distinguish a target from a vertex, but the vertices and the targets are otherwise indistinguishable, i.e., all vertices are visually identical and all targets are visually identical. Among the targets or the vertices it is only the cyclic order in which the robot sees the features that distinguishes them from each other. We assume that the ordering is always consistent, which we take, without loss of generality, to be counterclockwise. We model such a discrete vision by a point identification vector (piv), which is a vector defined by the cyclically ordered list of targets and polygon vertices along with some possible extra information they might carry (like the presence of a pebble) that are visible from the current robot's position. A pebble is a device that can be put on a position where a robot can stop (and recollected later) and is visible from afar. The entry of the piv corresponding to the vertex or target where the pebble is on will evidence this. Sitting at a vertex of $P$, we assume that the cyclic order of the visible points (vertices and targets) starts with the neighboring vertex, i.e., the first component of the piv always represents the neighboring vertex. For the robot located on a target, we make no assumption about the first component of the robot's piv - it is chosen by an adversary.

Moreover, the robot can see the edges of the polygon. This is modeled by a combinatorial visibility vector (cvv), a binary vector of length $k$ whose $i$-th bit encodes whether there is an edge between vertex $i-1$ and vertex $i$ of the $k$ vertices visible from the robot's position. See Fig. 1 for illustration. 


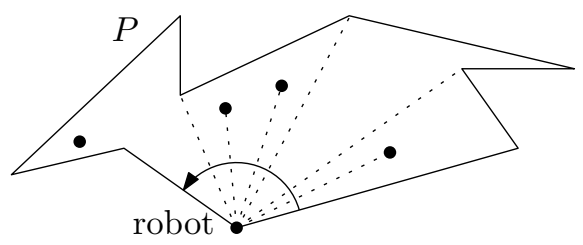

Figure 1: An illustration of a point identification vector (piv) and a combinatorial visibility vector (cvv) in polygon $P$ (with 4 targets); piv is $(v, t, v, v, t, t, v, v)$ (where $t$ stands for target and $v$ for vertex) and crv is $(1,1,0,1,0,1)$.

The robot has no other sensing capability, and in particular has no information about distances, angles, or world coordinates. This also motivates our simplistic model of the robot's movement. Roughly speaking, the robot can pick a direction based on its sensing system and move in that direction until the environment prevents the robot to go any further. The robot can sense the environment only when it is not moving. The destination of a robot's movement is either a target (only possible in the friendly scenario) or a visible vertex of $P$. We allow a robot a very special move to a vertex which is the endpoint of a partially visible edge of $P$, where an edge is said to be partially visible if only a proper subset of it is visible from the robot's current position (see Fig. 2). Note that in a simply-connected polygon this implies that at least one of its endpoints is hidden from the robot. In this case, the robot chooses one of the two endpoints (left or right) of a partially visible edge $e$. It reaches that vertex by walking straight to a visible point of $e$ chosen by an adversary and finally moving along the edge $e$ without stopping. This very special move will be used only in a 2-approximation algorithm (see Section 3.2).

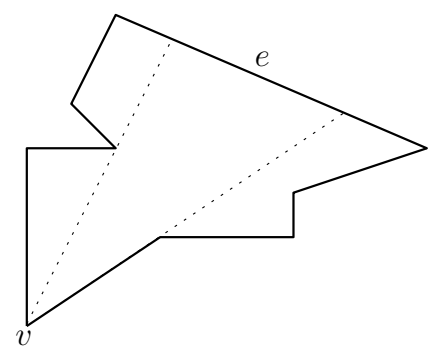

Figure 2: The edge $e$ is partially visible from $v$.

This model does not allow a robot to decide whether a given vertex is convex or reflex [1]. However we endow the robots with a special sensor that enables it to distinguish the type of the angle. This capability is important in the section about Hamiltonian triangulations. Obviously, a robot that can walk along edge-extensions can distinguish reflex angles from convex angles.

The robots model simple and small mobile sensors, which possess a lowresolution camera and limited computational power, which allows the robots to 
perform only simple image processing tasks, such as finding areas of substantial light changes. The limited power and equipment prevents learning anything more, like distances, angles, etc., and thus a binary sensing reflects appropriately what robots sense. The robots shall explore an unknown environment, which is physically bounded, such as buildings (with walls), or streets of a city. Thus we naturally arrive at the model with a polygon $P$ and discrete sensing via piv and crv.

Due to these unsophisticated vision and motion primitives, seemingly easy tasks become difficult in this model. For instance, a robot sitting at a vertex $u$ can specify a visible vertex $v$ by its index in the cvv of $u$. However, if the robot moves from $u$ to $v$, it is not possible in general to recover the position of $u$ with respect to $v$. A way to circumvent this issue is to mark $u$ with a pebble before moving to $v$. A pebble is visually distinguishable from vertices and targets. If no other pebbles are visible from $v$, the position of $u$ can be recovered. For a detailed discussion of the implications of this minimalistic model, see [1].

We are interested in how the robots can solve various environment exploration tasks and what limitations are implied by our simplistic assumptions on robots' capabilities. In this paper we consider the problem of determining the number of targets in an unknown polygon $P$. Throughout this paper we refer to this problem as the counting problem. By $n$ we denote the number of vertices of $P$ and by $m$ the number of targets therein. For simplicity we assume that the targets and polygon vertices are in a general position, i.e., no three points are collinear. In this paper we consider two different scenarios to model two basic classes of applications. In the friendly environment, the robot is allowed to walk to any target. In the hostile environment, the robot is not allowed to walk to targets. This scenario models the situation where a target represents an unsafe entity and coming into an imminent closeness to targets is dangerous.

For the friendly scenario we show that a single robot with two pebbles (markers) can count the number of targets in any polygon $P$. In contrast, we show that in the hostile scenario, the robots cannot count the targets in general. However, we identify a class of polygons where the counting problem is always solvable and we show that a robot can resolve whether it is located in such a polygon. Requiring the robots to count targets only from afar in general polygons is a more complicated problem, and we must endow the robots with some additional capabilities as anticipated before. Surprisingly, we show that these additional capabilities are quite minor, yet subtle. In fact, we consider two possible models, and show their implications on our problem. We consider robots that can walk along edge or diagonal extensions, i.e., if a robot picks a visible vertex $u$ as the direction of its walk, the robot can continue its walk in the same direction after it reaches $u$, if there is no polygonal edge to prevent it. In the second model we consider one additional global direction (think of "north") in which the robot can walk from any vertex of $P$. This types of motions enable a robot to specify consistently new points on the boundary of the polygon. Consequently we can show the a robot can solve the counting problem in both models.

We are interested in deterministic algorithms and their worst-case analysis, which we express in terms of the number of steps (movements) of robots and 
in the amount of used memory. We work with word-memory units, where one word of memory has $\Theta(\log (\max \{m, n\}))$ bits.

We are also interested in approximation algorithms, i.e., in algorithms that deliver a (provably good) estimate on the number of targets. Further, we look for estimates that are never smaller than the actual number of targets. We say that an algorithm is a $\rho$-approximation for the counting problem if for the setting with $m$ targets, $m \in \mathbb{N}$, the algorithm estimates the number of targets by $z$, for which $m \leq z \leq \rho \cdot m$.

To demonstrate the notion of approximation and to justify our sensing model we illustrate that for the following weaker sensing model no non-trivial approximation exists: consider the sensing of the vertices in the same way as we defined before, but consider the sensing of the targets only by their presence, i.e., not interleaved with the vertices. Thus, the only information the robot gets is the number of visible targets (but not their ordering within the vertices of $P$ ). Consider Fig. 3. It depicts two different scenarios, one scenario with $m=1$ target and the second scenario with $m=n / 3$ targets. In both scenarios the robot senses from every vertex exactly one target and therefore cannot distinguish the scenarios. Hence, for this simple sensing model no approximation algorithm can guarantee a ratio better than $n / 3$.

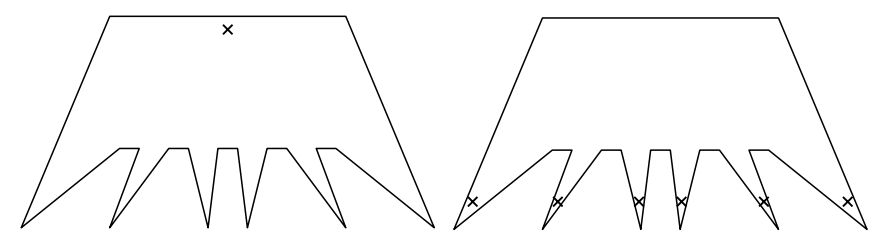

Figure 3: If a robot only senses the number of targets then the number of targets cannot be approximated within $o(n)$.

Related Work. Suri et al. [1] considered simple robots with combinatorial sensing of the environment and investigated some elementary questions of what information about the topology of the environment can be deduced by simple robots. In our paper, we consider the same robots with the additional capability to walk to the endpoints of partially visible edges, and we enlarge the complexity of the environment by adding the targets into the environment. Although the robots are strongly limited in capabilities, it is shown in [1] that the robots can solve non-trivial tasks. A robot cannot decide whether a vertex is convex, but can decide whether the polygon is convex. Also, a robot cannot decide which is the outer boundary of a multiply-connected polygon $P$, although it can discover and count all the boundary components in $P$. Furthermore, a robot with one pebble can build a mental map of the vertices of any (simply- or multiply-connected) polygon $P$ in $O\left(n^{3}\right)$ steps and with $O(n)$ memory, which allows the robot to navigate from any vertex $i$ to any vertex $j$. The navigation result is an important building block in our paper. Further, the paper shows 
that the robot can compute a triangulation of $P$ and solve a distributed version of the famous Art Gallery Problem [2] with $\lfloor n / 3\rfloor$ guarding positions. The impossibility results in [1] only exploit the primitiveness of the sensing system, hence they carry over easily to the model presented here.

Combinatorial geometric reasoning is used in many motion planning and exploration tasks in robotics [3, 4]. Minimalistic models of robots has been previously studied in $[5,6,7,8]$. However, the nature of problems investigated in our paper does not seem to be addressed in the past. The aforementioned papers deal with different problems such as navigation and pursuit evasion $[6,7,8]$, and not with recognition of important points (targets) in the environment. Learning about the geometrical nature of the environment is the problem studied in [5], where the environment is not a polygon, and it contains labeled features, which allows sensors to distinguish these landmarks.

\section{The Friendly Environment}

In this section we show that in a friendly environment a robot with two pebbles can count the targets in any simply- or multiply connected polygon.

We consider simply-connected polygons first. In the beginning the robot counts $n$, the number of vertices of the polygon: the robot leaves a pebble on the starting vertex and walks around the polygon's boundary, counting the vertices until it returns to the pebble. Let $1,2, \ldots, n$ denote the vertices of the polygon, ordered in the counterclockwise direction, starting at the robot's initial position.

The idea of the algorithm is to go to every vertex $i, i=1,2, \ldots, n$, and count the targets that are visible from $i$ and that are not visible from any vertex $j$, $j<i$. We call these targets newly visible at vertex $i$. Thus, the robot can go through vertices $i=1,2, \ldots, n$ and sum up all newly visible targets. Clearly, no target will be counted twice, and therefore the resulting sum is the total number of targets.

We now describe how the robot can identify whether a target is newly visible. Being at vertex $i$, the robot wants to identify whether a $k$-th target in its visibility vector is newly visible. The robot goes to the target, leaves a pebble there, and checks for every vertex $j<i$, whether the pebble is visible from $j$. The navigation from the target back to the vertex $i$ can be done by leaving the second pebble at $i$ and checking the position of $i$ in the visibility vector of the target. Obviously, the target is newly visible if and only if the pebble is not visible from any vertex $j, j<i$. Overall, the robot needs two pebbles and a constant number of memory words (to remember the number of vertices, the current position $i$, the position $j$ and the position $k$ of the considered target at $i$, and to mark the newly visible targets in the visibility vector of vertex $i$ ). Hence, in $2 i$ steps we can check whether a target visible from the $i$-th vertex is newly visible. To check all targets at position $i$ we need at most $2 m i$ steps. Thus, the robot needs $O\left(m n^{2}\right)$ steps to count the targets in $P$.

If the time is crucial, one can achieve a $O(m n)$ number of steps at the 
expense of used memory. For each vertex $i$ the robot maintains the piv with the additional information stating whether a given target is newly visible. In the beginning, every target in the piv is marked as newly visible. Then for every vertex $i$ the robot marks each newly visible target with a pebble and walks around the boundary towards vertex $n$ and at every vertex $j$, if the robot sees the pebble, it marks the corresponding bit in the bit array of vertex $j$ as not newly visible. Thus, the robot walks $m$ times around the boundary (for each target it walks exactly once and at most $n$ steps), resulting into $O(m n)$ steps of the robot. The robot needs $O(\mathrm{~nm})$ memory.

Theorem 1 In the friendly environment a robot with two pebbles can count the targets in a simply-connected polygon in $O\left(m n^{2}\right)$ steps and with $O(1)$ memory, or in $O(\mathrm{mn})$ steps and with $O(\mathrm{~nm})$ memory.

The result can be easily extended to polygons with holes (multiply-connected polygons), if we can navigate through the vertices in a consistent way. In [1], a navigation in an arbitrary multiply connected polygon was demonstrated with a robot with one pebble in a polynomial number of steps and with polynomial space. Our robot has all the capabilities of the robot described there, therefore the robot can first compute the navigation instructions, which are then stored in the robot's memory. Alternatively, we can use an additional, globally distinguishable pebble and perform the vertex navigation on the fly.

Theorem 2 In the friendly environment a robot with two pebbles can count the targets in any polygon in polynomially many steps and with polynomial memory.

\section{Hostile Environment}

After solving the counting problem in the scenario where robots can walk to targets, we consider now the scenario where robots walk only to vertices of $P$.

\subsection{Partition of the Polygon and Counting}

\section{General Idea}

The algorithms are based on the idea of partitioning the polygon into triangles and counting the targets in these triangles exactly. To illustrate the idea, consider a partition of $P$ into triangles having their vertices on the boundary of $P$ with the property that every triangle has at least one side on the boundary of $P$. We call such a triangle a baseline triangle, and each edge of the triangle that lies on the boundary of $P$ a baseline edge. A partition of a polygon into baseline triangles is called a baseline triangulation. We might want to require that the baseline triangles of a baseline triangulation are triangles in the classical sense, i.e., specified only by vertices of $P$ - these are called baseline vertex triangles (or only vertex triangles, if we do not insist on the baseline property). A triangulation into baseline vertex triangles is called a Hamiltonian triangulation [9], as 


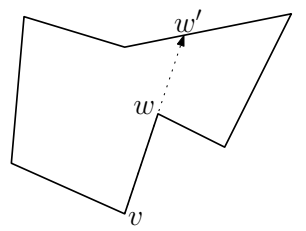

Figure 4: At $v$, a robot chooses $v w$ as the direction of the robot's walk. After reaching $w$, it can continue in the same direction until it hits the boundary at point $w^{\prime}$.

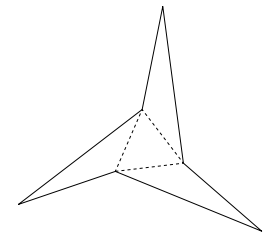

Figure 5: A polygon and its unique triangulation with triangles specified solely by vertices of the polygon. The triangulation is depicted by dashed lines.

its dual is a path. ${ }^{1}$ Unfortunately, a Hamiltonian triangulation does not always exist, see Fig. 5. We call a polygon that admits a Hamiltonian triangulation a Hamiltonian polygon.

Either of the two robot enhancements which we have introduced allows robots to use additional points of the boundary of $P$ in a baseline triangulation.

In the case of a baseline triangulation a robot can count the targets with the following algorithm. For every baseline triangle the robot moves to a vertex of the triangle (recall that this might not be a vertex of the polygon) opposite to a baseline edge, and counts the targets that are visible between the two vertices of the edge. Clearly, in this way every target is counted exactly once. Hence, a general algorithm that allows a robot to solve the counting problem is composed of a procedure to produce a baseline triangulation and of a navigation scheme to account for every baseline triangle exactly once.

\section{Computing a Hamiltonian Triangulation}

Narasimhan [10] presents an algorithm that recognizes whether a polygon has a Hamiltonian triangulation and if so computes one. The algorithm can be adapted for a robot that can discern convex vertices from reflex vertices (which is not directly possible in our model [1]).

So, for the purpose of this section, we assume that a robot is endowed with a special sensor that lets the robot be able to distinguish convex from reflex vertices. We show in the following, how such a robot can resolve whether a polygon admits a Hamiltonian triangulation and exactly count the number of targets in that case. However, when the polygon is non-Hamiltonian, this approach does not give anything useful, whereas our scheme, given in the following section, works for general polygons.

Preliminaries Every Hamiltonian triangulation has two distinguished triangles $T_{1}$ and $T_{2}$ which correspond to the two end vertices of the dual path.

\footnotetext{
${ }^{1} \mathrm{~A}$ dual of a triangulation is a graph, where each triangle corresponds to one vertex and there is an edge between two vertices iff the two corresponding triangles share an edge in the triangulation.
} 
Subsequently a Hamiltonian polygon has at least two distinguished vertices $s$ and $t$ which are the opposite vertices with respect to the edges of $T_{1}, T_{2}$ that are diagonals in $P$. In a polygon, there might be only few pairs $(s, t)$ (called feasible pairs) that have the aforementioned characterization. A sequential algorithm that aims at building a Hamiltonian triangulation strongly depends on the knowledge of the positions of $s$ and $t$. From now on we assume that $(s, t)$ is given as a part of the input for the algorithm which either returns a Hamiltonian triangulation or a certificate that $(s, t)$ is not a feasible pair. In fact the same algorithm can try every possible pair of vertices until the right one is found that identifies a Hamiltonian triangulation (if at all). In total, this costs not more than a $O\left(n^{2}\right)$ factor loss in the number of steps. Alternatively, a robot with $O\left(n^{2}\right)$ memory and a pebble can compute the visibility graph of the polygon in $O\left(n^{2}\right)$ steps and execute the dynamic programming based algorithm proposed in [9] which yields the pair $(s, t)$ or proves that one does not exist.

Our exposition of walks in Hamiltonian polygons, including notation and terminology, follows [10] closely. We call a simply-connected polygon $P$ discretely straight walkable, if there is a pair of vertices $(s, t)$ that divides the polygonal boundary into two internally vertex-disjoint directed paths (from $s$ to $t$ ) $L$ and $R$ with the following property: given two robots, each moving on one of both paths monotonically from $s$ to $t,(1)$ the segment connecting them is always completely contained in the polygon and (2) they can reach the destination under the constraint that when one robot is moving, the other is stationary at a vertex. This is called a feasible walk. Trivially, a Hamiltonian polygon is discretely straight walkable and vice versa. For this reason we aim at a feasible walking scheme for two robots (one on each path) that move monotonically from $s$ to $t$. By picking the diagonals defined by the position of the two robots during a walk we get a Hamiltonian triangulation.

To ease the description, we denote by $p, p^{\prime}, \ldots$ the vertices of $L$ and by $q, q^{\prime}, \ldots$ the vertices of $R$. Without loss of generality, we state our claims with respect to the vertices of $L$. Further, we introduce a partial order on the boundary of $P$ : given two points $l_{1}$ and $l_{2}$ of the same path, $l_{1}$ is smaller than $l_{2}$, denoted by $l_{1}<l_{2}$, if $l_{1}$ precedes $l_{2}$ on the path from $s$ to $t$. For a vertex $p \in L$, $\mathcal{V}(p) \subset R$ is the set of vertices of $R$ visible from $p$. In the context of a discretely straight walkable polygon, we aim at determining a subset $\mathcal{I}(p) \subset \mathcal{V}(p)$ of possible walking partners of $p$, i.e., a set of vertices where a robot can move on without compromising the feasibility of the walk, provided that the other robot is on $p$. If we can determine the set of walking partners, the following algorithm succeeds. Here, we call $l o V(p)$ and $h i V(p)$ the smallest and the biggest vertices of $\mathcal{I}(p)$, respectively.

Greedy Hamiltonian Triangulation - Two robots start at $s$. The robots move alternatively as follows: the moving robot moves along its path $(L$ or $R$ ) until it reaches $h i V(v)$ where $v$ is the vertex where the other robot is currently on. Stop when $t$ is reached.

First, observe that the greedy algorithm fails in general if $\mathcal{I}(p)$ is a maximal 
interval of $\mathcal{V}(p)$ that includes the position of the other robot before its next walking phase (Fig. 6).

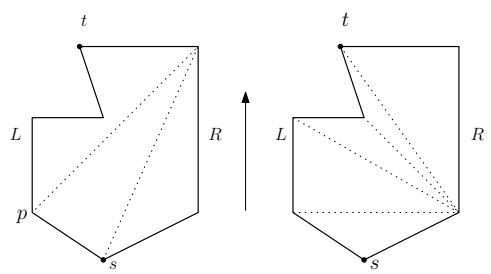

Figure 6: The greedy algorithm might fail, even though a feasible walk does exist. The left figure describes the situation when the robot moving on $R$ greedily proceeds. The right figure depicts a feasible Hamiltonian triangulation.

Characterization We want to determine an interval $\mathcal{I}(p)$ in such a way, that the following three properties hold:

- If $p<p^{\prime}$ then $l o V(p) \leq l o V\left(p^{\prime}\right)$ and $h i V(p) \leq h i V\left(p^{\prime}\right)$ (monotonicity).

- If $p$ and $p^{\prime}$ are adjacent, then $\mathcal{I}(p) \cap \mathcal{I}\left(p^{\prime}\right)$ contains at least a vertex (feasibility).

- If $q \in \mathcal{I}(p)$ then $p \in \mathcal{I}(q)$ (reciprocal visibility).

It is straightforward to see, that a scheme that produces a set of such walking partners enables a feasible walk in a Hamiltonian polygon. We recapitulate the main ideas of an algorithm [10] that yields a set with the above mentioned properties in the following.

To compactly describe the functions that determine the desired set of walking partners we need some more notation. For a point $p, \operatorname{Pred}(p)$ is the biggest vertex smaller that $p$ and $\operatorname{Succ}(p)$ is the smallest vertex bigger than $p$ (if these exist). Given a reflex vertex $p, \operatorname{Forw}(p)$ is the first intersection point between the polygon boundary and the edge-extension of the edge containing $\operatorname{Pred}(p)$ and $p$. Accordingly, $\operatorname{Backw}(p)$ is the first intersection point with the edge-extension of the edge containing $\operatorname{Succ}(p)$ and $p$. For an example see Fig. 7.

Fix a vertex $p \in L$ and consider the following functions:

$$
\begin{aligned}
h i P(p) & :=\min \{q \text { vertex of } R \mid L \ni \operatorname{Backw}(q)>p\} \\
h i S(p) & :=\min \left\{\operatorname{Forw}\left(p^{\prime}\right) \in R \mid p^{\prime} \in L, p^{\prime}>p\right\} \\
h i(p) & :=\min \{h i P(p), h i S(p), t\} \\
l o P(p) & :=\max \{q \text { vertex of } R \mid L \ni \operatorname{Forw}(q)<p\} \\
l o S(p) & :=\max \left\{\operatorname{Backw}\left(p^{\prime}\right) \in R \mid p^{\prime} \in L, p^{\prime}<p\right\} \\
l o(p) & :=\max \{l o P(p), l o S(p), s\}
\end{aligned}
$$




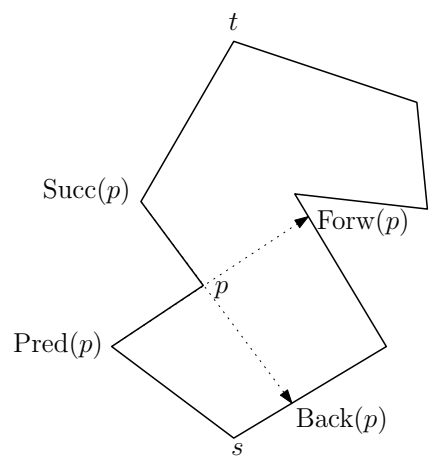

Figure 7: The functions $\operatorname{Backw}(p)$ and $\operatorname{Forw}(p)$ for a vertex $p$.

The main result of [10] is that a polygon is discretely straight walkable (and hence a Hamiltonian triangulation exists) if and only if the interval $[l o(p), h i(p)]$ contains at least one vertex of the polygon for every vertex $p$ (and accordingly for $q)$. Pick $\mathcal{I}(p)$ to be the maximal interval defined by vertices in $[l o(p), h i(p)]$. If we can show that a robot can compute $\mathcal{I}(p)$, then a single simple robot can easily exploit the idea of the aforementioned greedy counting algorithms and solve the counting problem in Hamiltonian polygons.

To this end we first state a useful observation. A fixed pebble on the vertex $s$ introduces a localization system for the robot: every vertex $p$ can be characterized by the path to which it belongs (which in turn, can be specified as the path on the right or left with respect to $s$ ), and by its distance to $S$ on this path, measured as the number of polygonal-edges between $s$ and $p$. Suppose for a moment that a robot is able to walk along edge-extensions and that it wants to compute the interval $[l o(p), h i(p)]$. To compute $h i S(p)$ and $l o S(p)$ it suffices to examine every single reflex vertex bigger (respectively, smaller) than $p$ : the robot walks along the corresponding edge-extension, it leaves a pebble at the hitting point and resolves whether the point is in the right path ( $R$ in this case) and whether it is smaller (respectively, bigger) than the current best. Similarly, $h i P(p)$ and $l o P(p)$ can be determined and thus $[l o(p), h i(p)]$. Actually, we are only interested in the vertices contained in $[l o(p), h i(p)]$. For this reason, the capability to walk along the edge-extensions is not essential, as the following Lemma will show.

Lemma 1 A simple robot can determine the (possibly empty) interval $\mathcal{I}(p)$ for every vertex $p$ of any simply-connected polygon.

Proof: We focus on $h i V(p)$, i.e., we consider just the functions $h i P(p)$ and $h i S(p)$ for a vertex $p$ (the other case is similar). Suppose that $P$ is discretely straight walkable with respect to the pair $(s, t)$, i.e., $\mathcal{I}(p)$ contains at least a vertex for every $p$. Further, assume that $h i(p)$ is determined by $h i S(p)$, that is, there is a vertex $p^{\prime}$ bigger than $p$ such that $\operatorname{Forw}\left(p^{\prime}\right)$ among those in 
$R$ is minimal. Let $q$ denote the first vertex of $R$ smaller than $\operatorname{Forw}\left(p^{\prime}\right)$, i.e., $q=\operatorname{Pred}\left(\operatorname{Forw}\left(p^{\prime}\right)\right)$. Clearly, $q$ is visible from $p^{\prime}$. We claim that $q$ is also visible from $\operatorname{Pred}\left(p^{\prime}\right)$. Then, $q$ is identified as the vertex that precedes $p^{\prime}$ in the visibility vector of $\operatorname{Pred}\left(p^{\prime}\right)$. This shows how to compute $\operatorname{Pred}\left(\operatorname{Forw}\left(p^{\prime}\right)\right)$, the candidate for $h i V(p)$, for any vertex $p^{\prime}$.

To prove that $q$ is visible from $\operatorname{Pred}\left(p^{\prime}\right)$, we look at all possibilities for an edge $e$ that might hide $q=\operatorname{Pred}\left(\operatorname{Forw}\left(p^{\prime}\right)\right)$ from $\operatorname{Pred}\left(p^{\prime}\right)$ (see Fig. 8). Obviously it is not possible that $e$ is defined by vertices bigger than $p^{\prime}$ or Forw $\left(p^{\prime}\right)$, respectively, because this intersects the line between $p^{\prime}$ and $h i S(p)$ and would have changed the point $\operatorname{Forw}\left(p^{\prime}\right)$. Similarly, if the edge $e$ had its endpoint smaller than $p^{\prime}$, then $\operatorname{Pred}\left(p^{\prime}\right)$ would not be visible from vertices in $R$, which is not possible. Finally, if $e$ has endpoints smaller than Forw $\left(p^{\prime}\right)$, there is a vertex $q^{\prime}$ smaller than Forw $\left(p^{\prime}\right)$ such that either $\operatorname{Backw}\left(q^{\prime}\right)$ is bigger than $p$ (unless one of the other two aforementioned cases occur) or $\operatorname{Succ}\left(q^{\prime}\right)$ is not visible from $L$. This shows that Forw $\left(p^{\prime}\right)$ does not correspond to $h i(p)$, a contradiction.

If $h i(p)$ is determined by $q=h i P(p)$, one can show by an analogous case study that the biggest endpoint of the edge hit by $\operatorname{Backw}(q)$ is visible from $\operatorname{Succ}(q)$ and thus $q$ can be computed from the visibility vector as well. The computation of $l o(p)$ can be handled in a similar way $(l o V(p)$ is required just to prove that $(s, t)$ is not a feasible pair).

Thus the robot can determine $h i V(p)$ by examining separately $h i P(p)$ and $h i S(p)$ and picking the smallest vertex (loV $(p)$ analogously). $h i S(p)$ is determined by examining the biggest vertex smaller than the point Forw $\left(p^{\prime}\right)$ for every reflex vertex $p^{\prime}>p$ in $L$ and comparing the corresponding distances to $s$. $h i P(p)$ is settled similarly, by going through the reflex vertices $q$ of $R$ and considering the smallest vertex bigger than the hitting point $\operatorname{Backw}(q)$.

If $P$ is not discretely straight walkable, the robot will find a vertex for which $h i V(p)$ is smaller than $l o V(p)$.

Note that for the algorithmic procedures described in the previous proof we do not require any additional pebble than the one for $s$. Further it is clear that we need only a constant number of memory words to perform every step and from step to step it is enough to maintain only the endpoints of the last picked diagonal. To compute every function for a fixed point $O\left(n^{2}\right)$ steps are sufficient, leading to a $O\left(n^{3}\right)$ algorithm. On the other hand, if the robot has $O(n)$ memory, it can directly compute every edge-extension first in $O\left(n^{2}\right)$ steps and subsequently find the Hamiltonian triangulation locally.

Theorem 3 Given a Hamiltonian polygon and a feasible $(s, t)$ pair, a simple robot which can distinguish reflex and convex vertices, with a pebble and $O(1)$ memory, can count the number of targets in $O\left(n^{3}\right)$ steps. If the robot has $O(n)$ memory, the task can be accomplished in $O\left(n^{2}\right)$ steps.

With the discussion from the beginning of this section, we get the following corollary. 

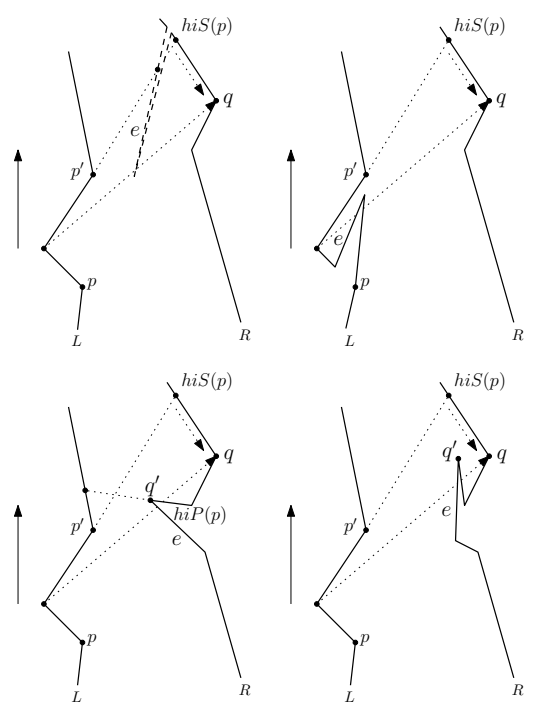

Figure 8: The four cases in the proof of Lemma 1

Corollary 4 Given a simply-connected polygon, a simple robot which can distinguish reflex and convex vertices, with a pebble and $O(1)$ memory, can count the number of targets or show that $P$ is not Hamiltonian in $O\left(n^{5}\right)$ steps. With $O(n)$ memory the complexity decreases to $O\left(n^{4}\right)$ steps and with $O\left(n^{2}\right)$ memory we have a further improvement to $O\left(n^{2}\right)$.

\subsection{Inapproximability and Approximation}

\section{Inapproximability.}

We show that the counting problem cannot be approximated within a factor $2-\varepsilon$, for any $\varepsilon>0$, even if the polygon $P$ is simply-connected. We start with a warm-up example to illustrate the idea. Consider the polygon in Fig. 9. The polygon consists of four spikes attached to the four sides of a rectangle. It depicts two scenarios with a different number of targets. In the first scenario there are 6 targets and in the second scenario there are 4 targets. Considering any vertex of the polygon, the vectors cvv and piv are the same in both scenarios. Hence, the robot cannot distinguish the two scenarios, which shows a lower-bound of $6 / 4=3 / 2$ for the approximation ratio.

This construction can be extended to a general-sized polygon, where $2 k$ spikes are attached to a regular $2 k$-gon, using $2 k$ and $4 k-2$ targets in two different scenarios, thus giving the desired inapproximability lower-bound of $2-\varepsilon$.

Theorem 5 The counting problem cannot be approximated within a factor $2-\varepsilon$, for any $\varepsilon>0$, even in a simply-connected polygon. 


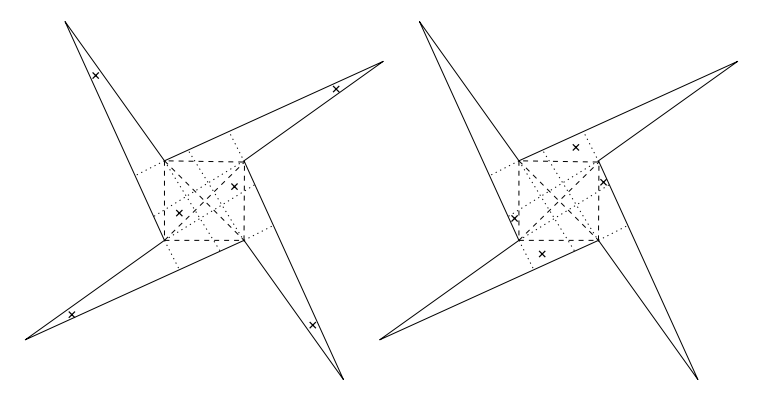

Figure 9: The counting problem cannot be approximated within a factor $3 / 2$.

Note that this inapproximability result relies only on the visibility limitations of the robots and not on their limited navigation capabilities.

Proof: We assume $n$ is even, i.e., $n=2 k$. The shape of the polygon is depicted in Fig. 10 and 11. The polygon consists of $n$ outer vertices $y_{1}, y_{2}, \ldots, y_{n}$ and $n$ inner vertices $x_{1}, x_{2}, \ldots, x_{n}$. It can be viewed as an $n$ gon, a regular polygon formed by vertices $x_{i}, i=1, \ldots, n$, connected on each side $x_{i}, x_{i+1}$ to a triangular spike $x_{i}, y_{i}, x_{i+1}$. Here and further in the text, the indices are to be understood in a cyclic fashion. The line $y_{i} x_{i}$ intersects the segment $x_{i+1} x_{i+2}$ in the middle. Thus, the visibility region of $y_{i}$, i.e., the cone of $y_{i}$ defined by lines $y_{i}, x_{i}$ and $y_{i}, x_{i+1}$, intersects the visibility regions of vertices $y_{i-1}$ and $y_{i+1}$, but not the visibility regions of other $y_{j} \mathrm{~s}$.

Observe first that a robot at vertex $y_{i}$ sees only two vertices of $P$, namely vertex $x_{i}$ and vertex $x_{i+1}$. Further, a robot sitting at vertex $x_{i}$ sees all vertices $x_{j}, j=1,2, \ldots, n$, and vertices $y_{i-1}$ and $y_{i}$.

The aim is to place the targets in a way that a robot sitting at vertex $y_{2 l+1}$ sees one target (the piv is $(0,1,0)$ ), and a robot sitting at vertex $y_{2 l}$ sees 2 targets (the piv is $(0,1,1,0)$ ). For a robot at vertex $x_{i}, i=1, \ldots, n$, we want the robot to see exactly 1 target between each two consecutive vertices of its piv, i.e., we want the piv to be $(0,1,0,1,0,1, \ldots, 0,1,0)$. Observe that the consecutive vertices of piv at vertex $x_{i}$ are $y_{i}, x_{i+1}, \ldots, x_{n}, x_{1}, \ldots, x_{i-1}, y_{i-1}$. We show how to achieve such visibility with two different number of targets. First we use only $n$ targets and then we use $2 n-2$ targets.

To place the $n$ targets we proceed as follows. We place one target into each triangle $y_{i}, x_{i}, x_{i+1}$. Observe that the triangle is divided into three parts by the lines $y_{i-1}, x_{i}$ and $y_{i-1}, x_{i-1}$. Let us label the parts $P_{1}, P_{2}$ and $P_{3}$, starting at a part containing the vertex $x_{i+1}$. Fig. 10 illustrates the partition. For odd $i$, we place the target into part $P_{2}$. For even $i$, we place the target into $P_{1}$. Observe now that a robot indeed sees one target from every vertex $y_{2 l+1}$ and two targets from every vertex $y_{2 l}$. Observe also that any vertex $x_{j}$ sees exactly one target between two consecutive vertices $x_{i}, x_{i+1}$, $i, i+1 \neq j$, because the parts $P_{1}$ and $P_{2}$ of triangle $y_{i}, x_{i}, x_{i+1}$ contain exactly one target and the parts are completely visible from $x_{j}$ within the segment 


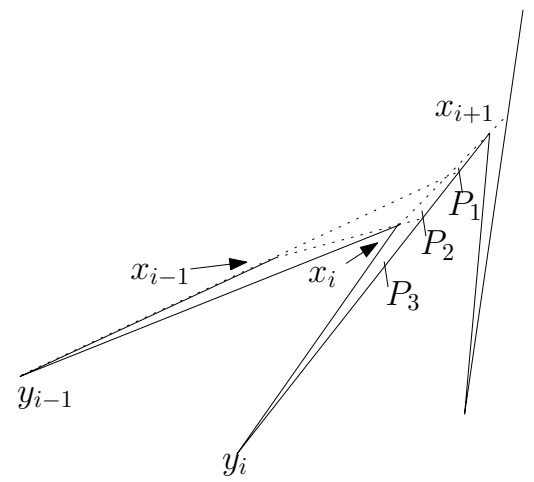

Figure 10: The partition of the triangle $y_{i}, x_{i}, x_{i+1}$ into three parts $P_{1}, P_{2}$ and $P_{3}$ by the lines $y_{i-1}, x_{i-1}$ and $y_{i-1}, x_{i}$.

$x_{i}, x_{i+1}$. There is also one target visible in the segment $y_{j}, x_{j+1}$ and in the segment $x_{j-1}, y_{j}$ which shows the claim for $n$ targets.

We now use $2 n-2$ targets in $P$ to achieve the same visibility configuration. First, we place one target into every triangle $x_{i}, y_{i}, x_{i+1}$ such that the target is visible only from vertices $x_{i}, y_{i}$ and $x_{i+1}$. This can be easily achieved when the target is placed very close to $y_{i}$. This leads to piv being $(0,1,0)$ at vertices $y_{i}$ and piv being $(0,1,0,0, \ldots, 0,0,1,0)$ at vertices $x_{i}$. The remaining $n-2$ targets are placed in the following way. For the presentation purposes we label the targets $t_{1}, \ldots, t_{n-2}$. Each target $t_{i}$ is placed close to vertex $x_{i}$ and in the cone $C_{i}$ of $x_{i}$ defined by the vertices $x_{n-1}, x_{n}$. More precisely, by placing $t_{i}$ close to $x_{i}$ we mean to place the target $t_{i}$ into the triangle $T_{i}:=x_{i-1}, x_{i}, x_{i+1}$. Observe now that for any placement of target $t_{i}$ into $C_{i} \cap T_{i}$ the piv of vertex $x_{i}$ is as desired, i.e., $(0,1,0,1,0, \ldots, 0,1,0)$. Indeed, for vertex $x_{i}, i \leq n-2$, the cone $C_{i}$ contains $t_{i}$ and thus the target is visible between $x_{n-1}$ and $x_{n}$. For every other cone of $x_{i}$ defined by vertices $x_{j}$ and $x_{j+1}$, the target $t_{j}$ lies in that cone. Also, for vertex $x_{n-1}$ the cone of $x_{n-1}$ defined by vertices $x_{i}$ and $x_{i+1}$ contains exactly one target, namely $t_{i+1}$. Similarly, the cone of vertex $x_{n}$ defined by vertices $x_{i}$ and $x_{i+1}$ contains exactly one target, namely $t_{i}$. To achieve the desired piv from the vertices $y_{i}$, we place each target $t_{i}$ within $T_{i}$ either to the left or to the right of line $y_{i-1}, x_{i-1}$. For $i-1=2 l$ we place $t_{i}$ to the right of the line $y_{i-1}, x_{i-1}$, so that $t_{i}$ is visible from $y_{i-1}$ (i.e., into the cone of $y_{i-1}$ defined by vertices $x_{i-1}$ and $x_{i}$ ). For $i-1=2 l+1$ we place $t_{i}$ to the left of line $y_{i-1}, x_{i-1}$, so that $t_{i}$ is not visible from $y_{i-1}$. It is easy to observe that for every vertex $y_{i}$, its piv is $(0,1,0)$ if $i=2 l+1$, and $(0,1,1,0)$ if $i=2 l$. A placement of $2 n-2$ targets into the polygon $P$ with $2 n$ vertices, where $n=12$, is depicted in Fig. 11. This ends the proof. 


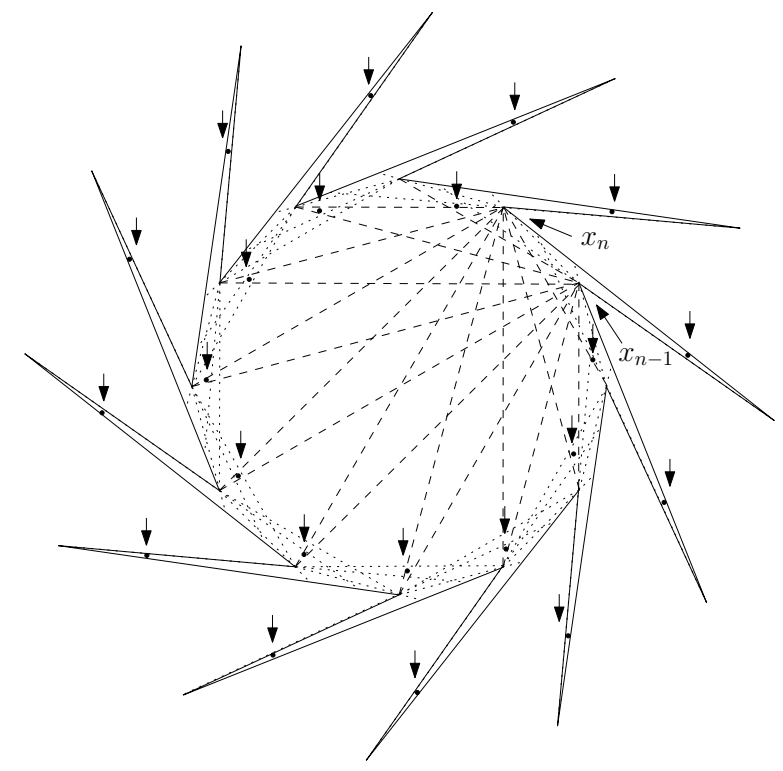

Figure 11: A placement of $2 n-2$ targets into the polygon $P$. The arrows indicate the position of the targets.

\section{Approximation.}

A Naive Approximation Since the counting problem cannot be solved optimally in general, it is natural to look for approximate solutions, i.e., for good estimates of $m$, the number of targets. Observe first that $m$ is at least the number of targets visible from any vertex of $P$. Let $m_{i}$ denote the number of targets that are visible from vertex $i$. We have $m \geq \max _{i} m_{i}$. On the other hand, clearly, $m \leq \sum_{i} m_{i}$. Since every target is visible from at least three vertices of $P$ (consider a triangulation of $P$ and the vertices of the triangle, in which the target lies), we have $m \leq \frac{1}{3} \sum_{i} m_{i}$. A robot can compute the sum $z=\sum_{i} m_{i}$ with one pebble that allows the robot to navigate through all vertices of $P$ (even with holes [1]). Obviously, reporting $\frac{1}{3} z$ as the estimate for the number of targets yields an $\frac{n}{3}$-approximation. Alternatively, if we denote by $k$ the number of vertices with non-zero $m_{i}$, the value $z / 3$ becomes a $\frac{k}{3}$-approximation.

Although the approximation is not sound at first sight (consider a convex polygon with a single target in it), it gives some insight into the complexity of the counting problem. Notice that the derived approximation ratio depends solely on the number of vertices $n$ (or on $k$, the number of vertices with a view on at least one target) and not on the number of targets. Hence, if $m$ grows in comparison to $n$ or $k$, the approximation ratio gets better. In other words, the approximation ratio does not grow with the number of targets, but is determined by the structure of the polygon (i.e., by $n$ ) and by the way how the targets are placed in this structure (i.e., by $k$ ). Moreover this approximation 
does not require the robots to be able to walk to the endpoints of partially visible edges, which is important in the next algorithm.

A 2-approximation Algorithm The previous result can be refined. We present in the following a 2-approximation algorithm for the counting problem. In light of the previous section, this approximation is the best possible.

The idea of the algorithm is to (recursively) partition a simply-connected polygon into discretely straight walkable subpolygons by adding suitable diagonals. In every subpolygon a Hamiltonian triangulation is built under the assumption that the added diagonals are actually edges in the subpolygon. By removing these diagonals we are left with the original polygon divided into baseline triangles, some of them extending into more than one of the mentioned subpolygons and hence overlapping. We will prove that every point of the polygonal area is covered by at most two baseline triangles, leading to the claimed approximation guarantee. To ease the description, we present the algorithm with two robots, but it will be easy to see that a single robot can simulate the algorithm.

First, a pebble is used to mark an origin vertex $s$, which introduces a localization system in the polygon $P$ as before. The two robots start a feasible walk from $s$ towards a determined destination vertex $t$ that is not yet determined, one on each path $(L$ and $R$ ). The robots move according to an arbitrary deterministic rule that maintains the visibility between the two robots. In the case that the walk cannot be extended without violating the visibility condition, one of the robots walks along the diagonal that connects its current vertex to the next vertex visible to the other robot. We call such a diagonal a gateway (see Fig. 12). They continue in this way until the robots meet at a common point. The gateways and edges that were traversed by the robots define a subpolygon $P_{1}$, which is discretely straight walkable by construction. The robots count the targets in all baseline triangles that were defined by the robots' walk.

All the gateways of $P_{1}$ define pockets of $P$ : a pocket is the subpolygon specified by the gateway and the portion of the boundary of $P$ that does not include the vertex from which the gateway was built. The pockets are partitioned recursively, but now the starting points $s_{L}, s_{R}$ and the destinations $t_{L}, t_{R}$ of the two robots' walks are fixed: given a pocket defined by a gateway $d$, the endpoints of $d$ represent $s_{L}$ and $s_{R}$, whereas $t_{L}, t_{R}$ are the endpoints of the edge $e$ partially visible between the endpoints of $d$ with respect to the robot's location $v$ where the gateway $d$ was built ${ }^{2}$. We say that $e$ is the shadow of $d$ with respect to $v$. To identify the endpoints of $e$, the robot sitting at $v$ walks to one of them and determines its position with respect to the localization system. The recursive partition of every pocket leads to a set of discretely straight-walkable polygons $P_{1}, \ldots, P_{k}$. When a robot counts the number of targets in a baseline triangle defined by a gateway, it possibly counts targets that are going to be counted again in the recursion of the corresponding pocket. Nevertheless, we

\footnotetext{
${ }^{2}$ Note that since the endpoints of $d$ are consecutive vertices in the cvv of $v, e$ is uniquely defined.
} 
claim that the algorithm counts every target at most twice and hence yields a 2-approximation.

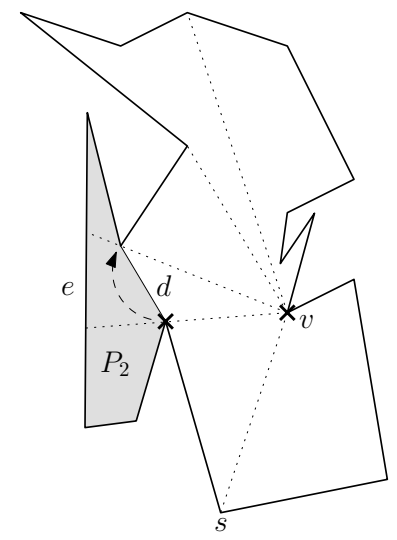

Figure 12: The picture depicts two robots (denoted by the crosses) that cannot continue their walk along the boundary. The vertex $v$ defines the gateway $d$, the pocket $P_{2}$ and the shadow $e$.

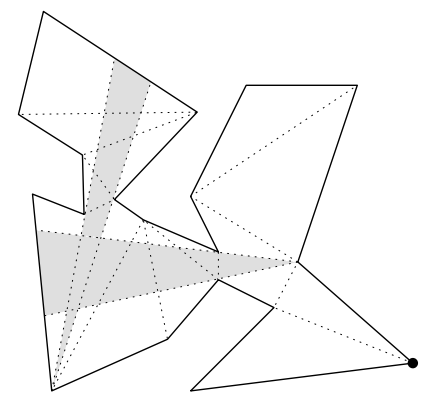

Figure 13: An example of the execution of the 2-approximation algorithm, started at the vertex marked by a dot. The shaded triangles are gateway triangles.

Theorem 6 A simple robot can approximate the number of targets within a factor two in any simply-connected polygon.

Proof: For simplicity, we prove the result for the original algorithm with two robots - the scenario with a single robot follows easily.

The algorithm induces as described a partition $\mathcal{P}$ of the polygon $P$ in discretely straight walkable subpolygons $P_{i}, 1 \leq i \leq k$. During the algorithm execution the robots build two types of triangles: vertex triangles (the baseline is an edge of $P$ ) and gateway triangles (the baseline is contained in an edge of $P$, the shadow of the corresponding gateway). The latter type are exactly the triangles that extend into more than one subpolygon $P_{i}$.

Consider a single subpolygon $P_{i}$ and the set of triangles generated exclusively by the recursion call in $P_{i}$. For a moment we restrict the gateway triangles by cutting off their portion not included in $P_{i}$. Since $P_{i}$ is discretely straight walkable, the triangles form a Hamiltonian triangulation of $P_{i}$. If we show that every subpolygon $P_{i}$ contains the portion of at most a single gateway triangle defined in an other subpolygon $P_{j}$, we obtain that every point contained in $P$ is covered by one or two triangles (neglecting the boundaries by the general position assumption) and the claim follows. 
So, consider the dual graph $T$ of the partition $\mathcal{P} .{ }^{3}$ Obviously, $T$ is a tree if $P$ is a simply-connected polygon. By following the algorithm execution in $P$, the edges of $T$ get an orientation. Every oriented edge $\left(p_{i} p_{j}\right)$ corresponds to a gateway triangle that has been specified by three vertices of the subpolygon $P_{i}$, but which has the baseline in the subpolygon $P_{j}$. Recall that the baseline of this gateway triangle is guaranteed to be an edge of $P$. So, by construction every gateway triangle traverses at most two subpolygons. Since $T$ is a tree, $P_{j}$ has at most a predecessor $P_{i}$ in $T$, hence there is at most a gateway triangle that might enter $P_{j}$.

\subsection{More Power to the Robots}

We have seen in the previous subsection that a simple robot cannot count the targets in a simply-connected polygon. We therefore look at possible enhancements of capabilities, which keep the robots as simple as possible and at the same time enable the robots to count the targets. We consider two such enhancements.

In the first one we allow the robots to walk along edge-extensions and diagonal-extensions, i.e., if a robot at vertex $v$ picks a vertex $w$ as the direction of the robot's walk, the robot is allowed to continue walking in the same direction after it reaches $w$, and it will stop only when it hits the boundary, at a point $w^{\prime}$. Fig. 4 illustrates this enhancement. The line $v w^{\prime}$ is called an edge-extension (diagonal-extension) if $v w$ is an edge (diagonal) of $P$. If we do not need to distinguish whether $v w^{\prime}$ is an edge- or diagonal-extension, we simply say that $v w^{\prime}$ is an extension. If a pebble is placed at $w^{\prime}$, it is then visible in the same way as a vertex of $P$, and therefore the robot can go there from any vertex visible to it. $w^{\prime}$ is then visually distinguishable from the other regular vertices of $P$, because it is marked with a pebble.

In the second enhancement one additional, global direction is introduced, in which a robot can move. Without loss of generality we assume that it is the direction of a vertical line going through the robot's position. For simplicity of presentation we assume that the polygon does not have vertical edges. On top of that we assume the robot can tell whether a visible point (a vertex or a target) is to the left or right of the vertical line, and whether it is above or below the robot, i.e., above or below the horizontal line going through the robot's position. Such an enhancement can be viewed as a navigation with compass. If a robot walks from a vertex $v$ in the vertical direction we say that it walks along the vertical extension of $v$.

For both enhancements we present algorithms that allow robots to count the number of targets inside the polygon $P$.

\footnotetext{
${ }^{3}$ Analogously as before, the dual graph of this partition is as follows: every subpolygon $P_{i}$ is a node $p_{i}$ of the graph $T$ and two nodes are connected iff the corresponding subpolygons share an edge (a gateway of $P$ ).
} 


\subsection{Walking along Edge- and Diagonal-Extensions}

In this section we consider robots that can walk along edge- and diagonalextensions. We show that such robots can partition any simply-connected polygon into baseline triangles, and thus can count the targets.

Consider a robot at a vertex $v$ of the polygon $P$. Let $v_{1}, v_{2}, \ldots, v_{i} \ldots$ denote the visible vertices from $v$, cyclically ordered in the counterclockwise direction. Observe that the lines $v v_{i}$ partition the visible part of $P$ into baseline triangles (all with a common point $v$ ), each with at least one baseline edge. See Fig. 14 for an illustration, where the triangles $v w_{1} v_{2}, v v_{2} w_{2}, v v_{3} v_{4}$ and $v v_{4} v_{5}$ partition the visible part of $P$. Thus we can partition the visible part of $P$. Observe that the invisible part of $P$ is a set of disjoint simply-connected sub-polygons. In the example from Fig. 14 the sub-polygons $P_{1}$ and $P_{2}$ form the invisible part of $P$. We call such a sub-polygon a pocket of $P$. Observe that a pocket is created by a line which is an edge-extension or a diagonal-extension. Applying a recursive partitioning approach on the pockets, we create a partition of $P$ into triangles with at least one edge on the boundary of $P$ (see Fig. 15). Let $T$ denote this triangulation.

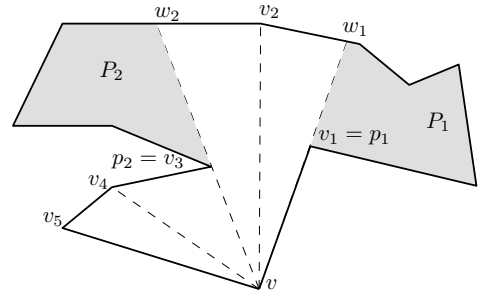

Figure 14: The extensions of a vertex $v$ define baseline triangles and pockets of $v$.

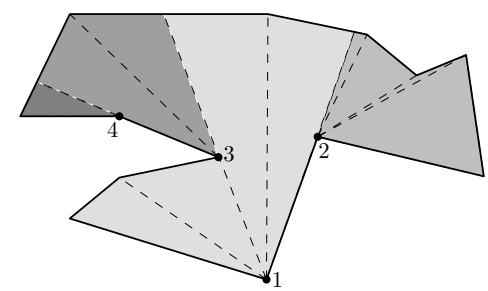

Figure 15: The resulting baseline triangulation of the algorithm and the visited pockets (grey). The labeled dots show the order of the recursion calles.

The main idea of the algorithm is to count all targets from the robot's position $v$ and then proceed recursively in the corresponding pockets of the polygon, thus navigating through $T$ and counting the targets in the triangles of $T$. We begin with a high-level description. For a vertex $v$ let $P_{1}, \ldots, P_{\ell}$ denote pockets of $P$ defined by all extensions originating at $v$. Let $p_{i}, i=1, \ldots, \ell$, denote the visible vertex whose extension defines $P_{i}$. Let $w_{i}$ be the point of $P$ for which $v w_{i}$ is the extension of $v p_{i}$.

\section{Counting in Simply-connected Polygons}

1. Count all the targets that are visible from the robot's position at vertex $v$.

2. Put a pebble at $v$ and remember the position of $v$ in the respective piv of every vertex $p_{i}$ and of every point $w_{i}$. 
3. Recursively count the targets in $P_{i}, i=1, \ldots, \ell$, by marking the point $w_{i}$ with a pebble and going to $p_{i}$.

When a robot walks to vertex $p_{i}$ to start a recursive call for pocket $P_{i}$, it first checks the position of the pebble that marks the point $w_{i}$. Next the robot determines which vertices (and targets) visible from $p_{i}$ belong to pocket $P_{i}$. Let $k$ be the number of vertices (including $w_{i}$ ) and targets visible from $p_{i}$. Let $h$ be the index of $w_{i}$ in the piv of vertex $p_{i}$. If pocket $P_{i}$ lies to the right of $p_{i} w_{i}$, then $P_{i}$ contains the vertices and targets from the piv of $p_{i}$ with index $1,2, \ldots, h$. If pocket $P_{i}$ lies to the left of $p_{i} w_{i}$, then $P_{i}$ contains the vertices and targets from the piv of $p_{i}$ with index $h, h+1, \ldots, k$. Observe that $P_{i}$ lies to the right of $p_{i} w_{i}$ if and only if $p_{i}$ is the first end-point of the diagonal in piv of vertex $v$.

The robot at vertex $p_{i}$ knows which part of its piv represents the sub-polygon $P_{i}$ and it can therefore perform the same steps of the Counting in Simplyconnected Polygons algorithm on the pocket $P_{i}$ only. Before that, the pebble from $w_{i}$ is collected as it is no longer needed. When the robot finishes the counting in $P_{i}$ it returns to the vertex $v$ (using the stored navigation information) and continues there.

Theorem 7 A robot with one pebble, able to walk along extensions, can count the number of targets in a simply-connected polygon of $n$ vertices in $O(n)$ steps with $O(n)$ memory.

Proof: Let $T$ be the baseline triangulation of $P$ produced by the algorithm. The triangles of $T$ are defined by vertices of $P$ and intersection points between polygonal edges and extensions. Observe first that the algorithm provides a consistent navigation scheme through $T$. Thus every target is counted exactly once. Note that the dual of $T$ is a tree. Every triangle of $T$ (except the triangle built as first) is specified by (a portion of) an edge of a neighboring triangle and by two vertices of the polygon: one is the vertex where the robot sits on and the other specifies the second non-baseline edge of the triangle. These two vertices define distinct edges or diagonals of $P$. Since a polygon has exactly $n$ edges and $n-3$ diagonals, $T$ has $O(n)$ vertices, which is also the number of steps of the algorithm (since the robot spends a constant number of steps in every triangle of $T$ ). The robot stores the necessary information to return from a recursive call - the predecessor $v$ of every vertex $p_{i}$. Hence, $O(n)$ memory is sufficient.

\subsection{Walking with a Compass}

In this section we consider in addition one fixed direction in which the robot can move. Without loss of generality, we assume that a robot sitting at a vertex can, additionally to moving to all visible vertices, move also along the vertical line going through the robot's position.

We present an algorithm that computes a baseline triangulation in any simply- or multiply-connected polygon and navigates the robot such that each triangle is considered for counting exactly once and thus it allows the robot 
to count the number of targets in the polygon. To simplify the presentation we first use an arbitrary number of pebbles - we show later how to use only a constant number of pebbles.

The key observation is that all the vertical extensions from vertices of a polygon $P$ partition the polygon into baseline triangles and convex quadrilaterals for which two opposite sides are on the boundary of $P$ (Fig. 16). Each quadrilateral can be subsequently partitioned into two baseline triangles (by picking a diagonal as the common boundary of the triangles).

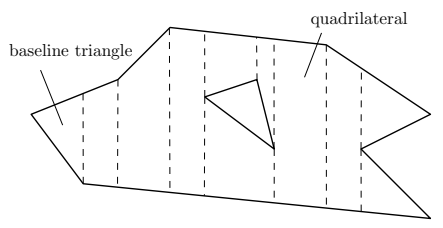

Figure 16: A multi-connected polygon with its partition by vertical extensions.

Hence, using at most $2 n$ pebbles, the robot can mark every end-point of every vertical extension which then imposes a baseline triangulation. This can be done by visiting every vertex of $P$ (using one pebble) [1]. To count every target exactly once, the robot goes through every vertex or pebble $p$ and considers only triangles lying above $p$ and on its right (if any). Since every triangle has one vertical side, the robot can always reach the opposite vertex of the baseline side in one step and count the targets in the triangle, and return back.

We now show how to reduce the number of used pebbles at the cost of an increased number of steps. The robot does not mark all the quadrilaterals at once, but one by one. Let us call an endpoint of a vertical extension a $q$-node. We show how to navigate through all the vertices and $q$-nodes in a consistent way. We begin with the navigation through vertices of $P$ from [1] (the navigation can be computed in $O\left(n^{3}\right)$ steps with $O(n)$ memory), where every edge of $P$ is visited exactly once. If a robot moves in this navigation along a polygonal edge $u v$, we compute all the $q$-nodes lying on this edge and before the robot moves to $v$ it visits all the $q$-nodes in the order of increasing distance from $u$.

Let us consider the situation where the robot is at a point $p$ (a vertex $u$ or a $q$-node) of the edge $u v$ and it wants to move to the next $q$-node. The robot can find the next $q$-node by sequentially creating all $q$-nodes (by going to every vertex of $P$ ) and checking which one lies on the edge $u v$ and closest to $p$. Specifically, using a pebble the robot marks the initial position $p$. The next pebble is used to mark the so-far closest $q$-node on the edge $u v$. The robot goes through every vertex $w$ of $P$ and creates $q$-nodes lying on the vertical extensions of $w$. For every such $q$-node the robot checks whether it lies on the edge $u v$ and whether it is closer to $u$ than the current best. The two pebbles make this operation easy for the robot.

Theorem 8 A robot with 2 pebbles, able to walk along vertical extensions, can count the number of targets in a polygon with $n$ vertices in $O\left(n^{3}\right)$ steps and with 
$O(n)$ memory.

\section{Conclusions}

We considered a minimalistic computational framework of mobile sensors - simple robots, whose visibility-based sensing reflects just the combinatorial character of the environment. We investigated their capabilities on the problem of counting points of interest (targets) in a polygon $P$ and considered two scenarios. We have shown that in the friendly environment the robots can count the targets using two pebbles. In the hostile environment the robots cannot count the targets and they cannot even approximate the number of targets by a multiplicative factor less than 2 . We have looked at possible minimum extensions of the robots' capabilities that allow to count targets. We have considered two such extensions - walking along edge- and diagonal-extensions, and walking with compass.

We have not answered all interesting questions and many of these remain open for the future research. For example, what is the best approximation ratio of the problem? Is the lower bound tight or is there a better approximation algorithm? What is the inherent power of pebbles: can we do anything without them? Are there simpler robots' enhancements that allow the robots to count the targets? Can a collaboration of more robots do better than a single robot?

\section{References}

[1] Suri, S., Vicari, E., Widmayer, P.: Simple robots with minimal sensing: From local visibility to global geometry. In: Proceedings of the TwentySecond National Conference on Artificial Intelligence and the Nineteenth Innovative Applications of Artificial Intelligence Conference, AAAI Press (2007) $1114-1120$

[2] Chvátal, V.: A combinatorial theorem in plane geometry. Journal of Combinatorial Theory 18 (1975) 39-41

[3] Latombe, J.C.: Robot Motion Planning. Kluwer Academic Publishers, Norwell, MA, USA (1991)

[4] LaValle, S.M.: Planning Algorithms. Cambridge University Press, Cambridge, U.K. (2006)

[5] Tovar, B., Freda, L., LaValle, S.M.: Using a robot to learn geometric information from permutations of landmarks. Contemporary Mathematics, to appear (2007)

[6] Yershova, A., Tovar, B., Ghrist, R., LaValle, S.M.: Bitbots: Simple robots solving complex tasks. In: Proceedings of the Twentieth National Conference on Artificial Intelligence and the Seventeenth Innovative Applications of Artificial Intelligence Conference. (2005) 1336-1341 
[7] Guibas, L.J., Latombe, J.C., LaValle, S.M., Lin, D., Motwani, R.: A visibility-based pursuit-evasion problem. International Journal of Computational Geometry and Applications 9 (1999) 471-494

[8] Sachs, S., Rajko, S., LaValle, S.M.: Visibility-based pursuit-evasion in an unknown planar environment. International Journal of Robotics Research 23 (2004) 3-26

[9] Arkin, E.M., Held, M., Mitchell, J.S.B., Skiena, S.: Hamiltonian triangulations for fast rendering. The Visual Computer 12 (1996) 429-444

[10] Narasimhan, G.: On Hamiltonian triangulations in simple polygons. International Journal of Computational Geometry and Applications 9 (1999) 261-275 